\title{
Linx
}

Revue des linguistes de l'université Paris X Nanterre

$11 \mid 1999$

Typologie des langues, universaux linguistiques

\section{Types de langues et types de constructions : le cas des corrélatives}

\section{Georges Rebuschi}

\section{(2) OpenEdition}

Journals

Édition électronique

URL : http://journals.openedition.org/linx/880

DOI : $10.4000 / \operatorname{lin} x .880$

ISSN : 2118-9692

Éditeur

Presses universitaires de Paris Nanterre

\section{Édition imprimée}

Date de publication : 1 décembre 1999

Pagination : 55-72

ISSN : 0246-8743

\section{Référence électronique}

Georges Rebuschi, «Types de langues et types de constructions : le cas des corrélatives », Linx [En ligne], 11 | 1999, mis en ligne le 03 juillet 2012, consulté le 19 avril 2019. URL : http:// journals.openedition.org/linx/880 ; DOI : 10.4000/linx.880

Ce document a été généré automatiquement le 19 avril 2019.

Département de Sciences du langage, Université Paris Ouest 


\title{
Types de langues et types de constructions : le cas des corrélatives
}

\author{
Georges Rebuschi
}

1.

1 Traditionnellement, la notion de type de langue correspond à l'idée que certaines propriétés des langues naturelles (usuellement frappantes au premier coup d'oeil) sont particulièrement importantes, v. par ex. la classification en langues isolantes, agglutinantes, synthétiques etc., ou encore l'idée que l'organisation nominativeaccusative ou absolutive-ergative des phrases transitives constitue un critère classificatoire fondamental ${ }^{1}$. Il s'agit là de "types " au sens fort, mais ceux qui les ont proposés ont rarement dit pourquoi ces propriétés étaient plus importantes que d'autres, si bien que les travaux de ce genre sont de plus en plus rares; d'un autre côté, l'usage du mot «type» peut être plus faible, auquel cas il s'agit simplement de propriétés différentielles non-hiérarchisées.

2 Parmi ces propriétés, on rencontre parfois la notion de possession ou non-possession d'une construction donnée, ce qui soulève un problème intéressant. L'enjeu est en effet le suivant. Pour les saussuriens, le signe se définit par la relation arbitraire qui unit la forme et le sens ; si le sens d'une expression complexe est calculable compositionnellement, c'est-à-dire sur la base exclusive du sens des morphèmes et lexèmes qu'elle contient et de leur agencement, il n'est pas arbitraire ${ }^{2}$, et cette expression n'a pas à être considérée comme un signe au sens strict. Pour la GGT, d'autre part, les constructions sont des artéfacts, des caractérisations descriptives approximatives ; il n'y a donc même pas lieu de se poser la question de savoir si un signe linguistique peut être complexe tout en restant un signe, c'est-à-dire un objet non seulement doté d'un signifiant, mais également d'un signifié : la possession d'une construction se ramène simplement à la valeur particulière de quelques paramètres qui, par leur interaction, permettent son émergence; mais cette position 
n'est évidemment tenable que si, à nouveau, on peut montrer que l'hypothèse compositionnelle l'est aussi. Dans le premier paradigme donc, la notion de possession d'une construction reste ouverte, mais le rasoir d'Occam devrait nous pousser à y renoncer dans la mesure du possible; mais dans le second paradigme, l'exigence de compositionnalité de l'interprétation n'est plus seulement souhaitable, mais absolument nécessaire, car si l'on renonce à la fois à la notion de construction et au principe de compositionnalité, les rapports entre forme syntaxique et sens ne relèvent plus de la recherche rationnelle.

\section{2.}

3 C'est dans cette perspective que je vais aborder la question des constructions corrélatives, définies pré-théoriquement comme des phrases complexes comprenant une protase à élément $k w-(q u-/ w h-. .$.$) et une apodose contenant un pronom de rappel ou corrélat, \pi$ ciaprès (j'emploierai « $\mathrm{PC}$ » pour me référer aux phrases corrélatives complexes complètes - uniquement) :

$(1)\left[\left[\left[_{\mathrm{CP}} k w_{-}^{-}\left[{ }_{\mathrm{IP}} \ldots t_{x} \ldots ..\right]\right] \ldots[\mathrm{IP} \ldots \pi \ldots]\right]\right.$

4 Ces constructions sont attestées dans les langues i.-e. anciennes (v. Haudry 1973), et dans diverses langues i.-e. contemporaines (langues slaves, hindi), mais on en trouve aussi en dehors du domaine i.-e.

\section{1.}

5 Dans certains cas, la contribution de la protase et de $\pi$ pris ensemble équivaut à celle d'un SN contenant une relative restrictive. Un tel SN est d'ailleurs parfois considéré comme primaire, la PC étant construite par transformation, comme dans la description scolaire suivante du grec ancien :

«Souvent le relatif précède le démonstratif, son antécédent logique. Le nom qui devrait accompagner le démonstratif est alors généralement placé par anticipation dans la proposition relative et s'accorde avec le pronom relatif. Ce nom ne prend pas l'article. » (Bizos 1966).

6 A part la remarque sur l'article, non pertinente, on a exactement la même chose en latin, comme l'indique (2a), paraphrasé comme (2b) par le grammairien, qui prend lui aussi cette dernière structure comme primaire (Gason 1962) :

\begin{tabular}{|l|l|l|l|l|l|}
\hline$(2)$ & a & Quas & & scripsisti & litteras, \\
\hline \hline & & quel[ACC,FM,PL] & & tu-écrivis & lettre[ACC,FM,PL] \\
\hline & & eae & mihi & joncundissimae & fuerunt \\
\hline & & elles & à-moi & très-agréables & ont-été \\
\hline & & 'La lettre que tu as écrite m'a fait grand plaisir' & \\
\hline
\end{tabular}


[Litteræ [quas scripsisti]] mihi joncundissimae fuerunt (id.)

7 Les ex. suivants illustrent le tour en hittite (Cooper 1979) et en hindi (Dayal 1996).

\begin{tabular}{|l|l|l|l|l|l|l|l|}
\hline (3) & aššer-ma-kan & kuiéš & dingir-mēš & $n$ - $\boldsymbol{a}$ š & ina e & Mezulla & pihhun \\
\hline \hline & restait-mais & quel & dieu-PL & et-eux & maison-à & M. & je-donnai \\
\hline & Lit. 'Quels dieux restaient, je les donnai au temple de Mezulla' \\
\hline
\end{tabular}

\begin{tabular}{|l|l|l|l|l|l|l|l|}
\hline$(4)$ & jo & larkii & kharii & hai, & vo & lambii & hai $^{3}$ \\
\hline & quelle & fille & debout & est & cette/elle & grande & est \\
\hline \hline
\end{tabular}

8 En dehors de l'i.-e., le bambara présente le même tour (Zribi-Hertz \& Hanne 1995) :

\begin{tabular}{|l|l|l|l|l|l|l|l|l|l|}
\hline (5) & Musa & ye & uru & $\min$ & sAn, & $n$ & ye & o & $y e$ \\
\hline & Moussa & $\mathrm{pf}$ & couteau & rel & acheter & $1 \mathrm{sG}$ & $\mathrm{pf}$ & lui & voir \\
\hline \multicolumn{7}{|l}{} \\
'J'ai vu le couteau que Moussa a acheté' \\
\hline
\end{tabular}

\section{2.}

9 Dans d'autres cas, la même structure globale peut donner lieu à une interprétation générique ou universelle. C'est à nouveau le cas du latin (6: Debeauvais), de l'ancien français ( 7 : Roland 2494), du russe $(8)^{4}$, ou, hors du domaine i.-e., du basque, typiquement dans ses variétés parlées en France ( $9:$ Mt 5,315).

\begin{tabular}{|l|l|l|}
\hline (6) & Ubi tyrannus est, ibi nulla est res publica \\
\hline & 'Où il y a un tyran, il n'existe plus de république' \\
\hline
\end{tabular}

(7) Ki mult est las, $\boldsymbol{i l}$ se dort cuntre tere ${ }^{6}$

\begin{tabular}{|l|l|l|l|l|l|}
\hline (8) & Kogo & žurjat, & togo & i & ljubjat \\
\hline \hline & qui-ACC & on-gronde & lui-ACC & et & on-aime \\
\hline
\end{tabular}


'On gronde ceux qu'on aime'

\begin{tabular}{|l|l|l|l|l|l|l|}
\hline (9) & Nork & ere & uzten & baitu & bere & emaztea \\
\hline & qui-ERG & même & laisse & C + AUX & sa & femme \\
\hline & eta & harek & eman & bezo & & ageria \\
\hline & et & lui-ERG & donne & qu'il-le-lui & & certificat \\
\hline & 'Quiconque abandonne sa femme, qu'il lui donne une attestation' & \\
\hline \hline
\end{tabular}

\section{3.}

10 Si l'on avait toujours la même interprétation globale, elle serait aisément redevable d'une analyse compositionnelle : le pronom $\pi$ de l'apodose pourrait s'interpréter comme un article défini employé intransitivement, c'est-à-dire comme un opérateur iota dont la restriction $R$ serait une variable de propriété (localement) libre, cf. Cooper (1979) ${ }^{7}$ :

(10) $\pi=>\lambda Q \cdot Q(1 x(R(x)))$

11 La protase, quant à elle, fournirait une valeur pour cette variable : la propriété pour $x$ d'être le couteau que Moussa a acheté en (5), ou d'être grondé en (8), etc. Mais c'est précisément parce que les interprétations ne sont pas les mêmes que l'on a un problème potentiel pour la compositionnalité : d'où vient en effet qu'en bambara, l'association protase $+\pi$ ne s'interprète que comme une relative spécifique, et qu'en basque ou en russe, seule l'interprétation générique soit normalement disponible? Si l'on peut donner une réponse à cette question, on aura évité de faire des PC des signes "saussuriens » complexes, et l'on pourra rechercher un micro-paramètre permettant à certaines langues de construire des protases corrélatives, sans plus.

12 Notons qu'à côté de ces deux extrêmes, on a encore une possibilité : en latin, c'est, semble-t-il, le temps et l'aspect qui sont déterminants ; si l'on en fait abstraction, les deux interprétations sont disponibles, v. (2) et (6). En hindi, selon Dayal, l'interprétation de base est typiquement définie, mais ces propriétés temporo-aspectuelles semblent aussi jouer un rôle : l'interprétation peut devenir universelle si le nom est au pluriel et si le temps est générique.

\section{3.}

13 Essayons donc de passer en revue quelques propriétés distinctives des structures globales dans les langues concernées afin de voir si elles peuvent être mises en rapport avec les différences d'interprétation notées à l'instant. 


\section{1.}

Certaines de ces langues permettent la duplication du $k w$-initial et par conséquent du corrélat $\pi$, et d'autres pas. Mais cette propriété semble indépendante de la question sémantique soulevée ici, puisque le hindi et le russe le permettent, que le basque le pouvait, mais ne le peut plus aujourd'hui :

\begin{tabular}{|c|c|c|c|c|c|}
\hline \multirow[t]{2}{*}{$(11)$} & \multicolumn{5}{|c|}{ hindi (Dayal, $1996:$ 197) } \\
\hline & jis & larkii-ne & jis larke-ke & saath & khelaa \\
\hline & $k w-$ & fille-ERG & $k w$-garçon & avec & joua \\
\hline & us-ne & us-ko & haraayaa & & \\
\hline & elle-ERG & lui-ACC & battit & & \\
\hline & \multicolumn{5}{|c|}{ lit. 'Quelle fille a joué avec quel garçon, elle l'a battu' } \\
\hline & \multicolumn{5}{|c|}{ = 'chaque fille qui a joué avec un garçon l'a battu's } \\
\hline
\end{tabular}

\begin{tabular}{|l|l|l|l|l|l|l|l|l|}
\hline (12) & \multicolumn{6}{|l|}{ russe (Izvorski 1996) } \\
\hline & Kto & kogo & ljubit, & tot & o & tom & i & govorit \\
\hline & qui-NOM & qui-Acc & aime & il & de & lui & et & parle \\
\hline & 'Celui qui aime quelqu'un en parle' \\
\hline
\end{tabular}

\begin{tabular}{|l|l|l|l|l|l|l|l|}
\hline (13) & \multicolumn{6}{|l|}{ basque ancien (Detchepare 1545 : I, 14)9 } \\
\hline & Nork & {$[$ zer } & hazi $]$ & erein & baitu & biltzen & dizi \\
\hline & qui & quelle & semence & semé & C'-Aux $^{\circ}$ & récolte & aux \\
\hline & 'On récolte le grain qu'on a semé' \\
\hline
\end{tabular}

\section{2.}

Le fait que l'élément $k w$ - soit un adjectif ou un déterminant ou, au contraire, corresponde à tout un $\mathrm{SN}$ ne semble pas non plus pertinent ; v. à cet égard non seulement les deux ex. latins supra, mais aussi la construction définie du hindi (11) ou du bambara (5), à comparer à la construction universelle russe suivante : 


\begin{tabular}{|l|l|l|l|l|l|l|l|}
\hline$(14)$ & Kakova & & zemlja, & takov & i & & xleb \\
\hline & comme & [est la] & terre, & tel & aussi & [est le] & pain \\
\hline
\end{tabular}

\section{3.}

Il existe dans certaines langues un tour marqué, dans lequel ce qui est normalement une protase corrélative est directement adjoint à une expression (pro)nominale :

\begin{tabular}{|l|l|l|l|l|l|l|l|l|l|l|}
\hline (15) & \multicolumn{6}{l|}{ hindi (Dayal p. 206) } \\
\hline & [jo & aaye & un-kaa & kaam $]$ & {$[$ jo } & gaye & un-ke & kaam]-se & behtar & hai \\
\hline & qui & vint & leur & travail & qui & partit & leur & travail- & meilleur & était \\
\hline
\end{tabular}

\begin{tabular}{|l|l|l|l|l|l|l|l|l|}
\hline (16) & \multicolumn{6}{l|}{ basque (Léon : Mc 10,40) ${ }^{11}$} \\
\hline & toki & hoik & [norentzat & ere & eginak & baitira eta & heienak] & dira \\
\hline \hline & siège & ces & pour-qui & même & faits & C $^{\circ}$-ils-sont et & à-eux & ils-sont \\
\hline
\end{tabular}

17 Mais comme ces ex. le montrent, cette tournure existe aussi bien en hindi, qui donne une interprétation typiquement définie, qu'en basque, qui ne fournit qu'une interprétation universelle ; il ne peut donc pas s'agir d'une propriété pertinente pour notre problème.

\section{4 .}

18 Comme on a pu le remarquer en (3), (8), (9) etc., ce qui est par ailleurs une conjonction de coordination apparaît souvent en tête d'apodose. On reviendra sur ce point plus loin mais son apparition dans l'ex. hittite, qui ne peut s'interpréter que de manière spécifique, et dans les ex. russes et basques, dont le sens est générique, montre que cette "particule » n'est pas pertinente pour l'opposition sémantique qui nous préoccupe.

\section{5.}

En russe, les pronoms corrélats, s'ils sont démonstratifs, apparaissent à l'initiale de l'apodose. C'est aussi une tendance en basque, qui utilise le mécanisme dit «V2 » comme stratégie de focalisation, en ce qu'un élément focalisé (comme un interrogatif) doit apparaître à l'initiale, immédiatement à gauche du verbe fléchi. Cependant, comme le 
note Izvorski (1996), ce positionnement privilégié en russe ne concerne pas les pronoms personnels, qui, pourtant, peuvent aussi matérialiser $\pi$. Qui plus est, l'ex. hittite (3) montre que cet ordre des mots peut aussi concerner les interprétations définies.

\section{6.}

L'élément $k w$ - des protases basques est souvent suivi de ere 'même/aussi', mais ce n'est pas toujours le cas, v. (13). En fait, cet élément (cf. ever en anglais) ne donne pas automatiquement lieu à une interprétation universelle, comme l'a noté Jacobson (1995) : il est compatible avec une interprétation spécifique ou plutôt « singulière ", marquant simplement que le locuteur est totalement incapable d'identifier le référent. De plus, le morphème hindi bhii a les mêmes propriétés, cf. (17), dont je conserve la trad. anglaise pour plus de transparence (Dayal $1996: 211$ ) :

\begin{tabular}{|l|l|l|l|l|l|l|l|l|l|}
\hline (17) & jo-bhii & larkii & vahaan & khaRi & hai & vo & Ravi-kii & dost & hai \\
\hline & whichever & girl & there & standing & is & she & Ravi's & friend & is \\
\hline \multicolumn{6}{|l}{} \\
\hline
\end{tabular}

\section{4.}

D'autres propriétés des PC sont plus étroitement reliées aux analyses spécifiques proposées par les différents auteurs, si bien qu'il est difficile, en l'absence de discussion des alternatives, de savoir si ces hypothèses sont les seules compatibles avec les données. Je signalerai cependant quelques points.

\section{1.}

Pour commencer, la position spécifique occupée par la protase est, selon les cas :

- adjointe à IP (Dayal : hindi)

- adjointe à $\mathrm{CP}$ (Izvorski : russe)

- en Spéc,CP (Z-H\&H : bambara)

Si $\pi$ est en Spéc,CP en basque (v.§ 3.5), il n’y a, dans cette langue, pas d'autre place pour la protase que l'adjonction à $\mathrm{CP}$, du moins dans une théorie qui ne démultiplie pas les têtes fonctionnelles. Quoi qu'il en soit, je n'imagine pas en quoi une localisation diversifiée de la protase pourrait jouer un rôle dans l'interprétation sémantique de $\pi$ et donc de la CP globale, puisqu'au niveau interprétatif, seule compte l'opposition entre position argumentale et position non-argumentale (cette dernière concernant les opérateurs).

\section{2.}

Les auteurs cités (Dayal, Izvorski, Z-H\&H) s'accordent à dire que la protase est générée dans la position où ils la situent. Cela semble être la solution la plus raisonnable (v. la note 3), et, à supposer que les structures illustrées en (15) et (16) puissent malgré tout être 
considérées comme servant d'input à un déplacement, le fait même que ces ex. soient respectivement en hindi et en basque montre que cette question n'est pas pertinente pour la différence sémantique qui nous occupe.

\section{3.}

Peut-il y avoir un apport spécifique du corrélat $\pi$ ? Ce n'est pas évident, car le fait que ces pronoms puissent aussi être des déterminants est clair dans les quatre langues suivantes : hindi, bambara, russe et basque. Z-H\&H proposent pour le bambara un type de relation anaphorique particulier entre la protase et ce corrélat; il est possible qu'il faille recourir à une telle analyse, étant donné les propriétés de $o$, mais cela ne devrait pas permettre de diviser les PC en deux types, car il est probable que l'opposition entre les deux types de pronoms russes ( $\$ 3.5$ ) relève de la même distinction. De plus, le fait que le basque labourdin ait connu un pronom particulier pour ce type de contexte aux $18^{\mathrm{e}}$ et $19^{\mathrm{e}}$ siècles, haina, (Rebuschi 1998, a, b) n'a rien changé aux propriétés de ses corrélatives.

\section{4 .}

Du côté de la contribution sémantique de la protase, la seule analyse précise dont je dispose est celle de Dayal, qui propose d'en faire un quantificateur généralisé (proche de l'opérateur iota russellien, mais employant des individus pluriels ${ }^{12}$ ):

\begin{tabular}{|l|l|}
\hline$(18)$ & $\lambda \mathrm{Q} \cdot \mathrm{Q}[\sigma \mathrm{x}(\mathrm{R} 1(\mathrm{x}) \& \mathrm{R} 2(\mathrm{x})]$ \\
\hline & $\begin{array}{l}\text { (où } \mathrm{R}_{1} \text { est la restriction apportée par le nom spécifié par le pronom relatif, et } \mathrm{R}_{2} \text {, le } \\
\text { prédicat de la protase) }\end{array}$ \\
\hline
\end{tabular}

Cette analyse pourrait sembler suspecte, dans la mesure où elle ressemble fortement à une simple stipulation (un déplacement de $k w$ - relatif construit une simple propriété), où elle complique à souhait la contribution sémantique de $\mathrm{C}^{\circ}$, qui devient tout à fait artificielle, et où elle force l'auteur à une gymnastique assez complexe dans le cas où l'interprétation n'est pas définie (\$ 2.3). Cependant, comme il existe des arguments tout à fait indépendants justifiant l'idée que les protases corrélatives sont en fait des thématisations, des reprises (cf. Gupta 1986, non cité par Dayal), l'interprétation des protases des PC comme des SN définis paraît inéluctable ${ }^{13}$. On verra au $\S 6$ comment résoudre cette question de manière moins artificielle.

\section{1 .}

Il existe cependant un facteur qui me semble exploitable pour dériver l'opposition sémantique entre les PC basques et slaves, d'une part, et les PC latines de l'autre. C'est le suivant: quand l'élément $k w$ - de la protase est clairement relatif, les structures sont ambiguës, seules les modifications temporo-aspectuelles de la phrase radicale pouvant forcer une interprétation ou une autre, v. (2) et (6). Par contre, dans des langues comme le russe ou le basque, les éléments $k w$-appartiennent au paradigme des interrogatifs (ce qui ne signifie évidemment pas que les protases soient interrogatives !) : le russe oppose 
ainsi l'interrogatif [+hn] kto au relatif kotoryj, et le basque, qui s'est débarrassé de ses relatifs au $19^{\mathrm{e}}$ siècle, opposait l'interrogatif [+hn] nor au relatif [ $\left.\pm \mathrm{hn}\right]$ zein - et, dans les deux cas, l'interprétation est normalement universelle.

\section{2.}

L'allemand littéraire (voire archaïque pour les tours en der...der) vient confirmer ce point de vue. Il a (ou avait) en effet deux types de constructions en $k w$ - disloquées à gauche, les unes, d'interprétation générique, introduites par un «relatif» (c'est la tradition qui parle) en $w$-, wer au nom. masc. sg. (la " relative » entière pouvant être reprise par un pronom-corrélat si le cas est le même, et devant l'être autrement), et les autres, d'interprétation spécifique, qui sont introduites par le vrai relatif en $d-$, der au nom. m.sg. ${ }^{14}$. Le premier cas est illustré par une traduction de Mc $4,9^{15}$ et par la dernière phrase du Tractatus de Wittgenstein, 1921 :

\begin{tabular}{|l|l|l|l|l|l|l|l|l|}
\hline (19) & Wer & Ohren & hat & $z u$ & hören, & der & höre! & (HS \\
\hline \hline & qui & oreilles & a & pour & entendre, & celui-là & entende & \\
\hline \multicolumn{6}{|l}{ Qui habet aures audiendi, audiat } \\
\hline
\end{tabular}

\begin{tabular}{|l|l|l|l|l|l|l|l|l|l|}
\hline (20) & Wovon & man & nicht & sprechen & kann, & darüber & muss & man & schweigen $^{16}$ \\
\hline & de-quoi & on & neg & parler & peut, & sur-ça & doit & on & se-taire \\
\hline
\end{tabular}

Le second cas est illustré par Mt 26,23 (ibid.):

\begin{tabular}{|l|l|l|l|l|l|l|l|l|l|}
\hline$(21)$ & Der & mit & mir & die & Hand & in & die & Schüssel & taucht, \\
\hline \hline & qui[REL] & avec & moi & la & main & dans & le & plat & trempa \\
\hline & der & wird & mich & verraten & & & & [HS] & \\
\hline & celui-là & aux & me & trahira & & & & & \\
\hline
\end{tabular}

31 Un autre fait vient confirmer que les protases en wer sont intrinsèquement universelles : si le relatif initial en der... peut se voir adjoindre le quantificateur universel alle 'tous', wer ne le peut pas, ce qui montre bien qu'il contient déjà l'information en question.

\section{3.}

On admet généralement que le mouvement d'un élément kw-relatif revient à opérer une abstraction- $\lambda$ sur la proposition à laquelle sa trace appartient : 


\begin{tabular}{|l|l|l|l|l|}
\hline$(22)$ & $\mathrm{a}$ & $k W^{-R E L}$ & $\left(\mathrm{C}^{\circ}\right)$ & {$\left[{ }_{\mathrm{IP}} \ldots \mathrm{ti} \ldots ..\right]$} \\
\hline & $\mathrm{b}$ & $=>$ & $\lambda x[\phi(x)]$ & \\
\hline
\end{tabular}

d'où l'interprétation des relatives restrictives ordinaires comme des propriétés qui, prises en extension, donnent un ensemble en intersection avec celui dénoté par le "nom-tête" antécédent. Il est naturel de traiter les protases corrélatives à relatif de la même manière. La question est donc de savoir pourquoi la substitution d'un $k w$-interrogatif ajoute une dimension d'universalité à l'interprétation globale de la PC.

Il faut mettre ce fait en relation avec la propriété qu'ont les interrogatifs introduisant des subordonnées d'exiger une réponse exhaustive. Considérons un modèle dans lequel trois individus, Pierre, Marie et Denis se seraient rendus à un certain endroit. Je puis poser la question (23a) à Jean et, s'il s'agit d'une véritable demande d'information (et non d'un test dont je connaîtrais la réponse), (23b) peut être considéré comme pragmatiquement convenable, même si (23c) peut suivre de ma part :

\begin{tabular}{|l|l|l|}
\hline$(23)$ & a & Qui est venu ? \\
\hline \hline & b & Marie et Denis. \\
\hline & c & C'est tout ? \\
\hline
\end{tabular}

Considérons maintenant (24) :

(24) Jean sait qui est venu.

Contrairement à (23b), dont on vient de voir que c'est une réponse relativement acceptable, (24) est simplement fausse si, moyennant le scénario décrit, l'énonciateur de (24) connaissait la vérité alors que Jean saurait seulement que Marie et Denis sont venus : on a donc une simple attente d'exhaustivité dans les questions directes, mais une exigence d'exhaustivité dans (24).

Or c'est une propriété des protases des PC que d'être non-indépendantes, comme le marque la présence du $\mathrm{C}^{\circ}$ bait- dans les ex. basques, et l'ordre des mots (avec verbe fléchi final) en allemand (en employant l'expression "non-indépendante", je veux souligner que ces protases, qui ne peuvent être énoncées seules, hormis ellipse suspensive bien entendu, ont les propriétés syntaxiques internes de propositions subordonnées, sans pour autant être dépendantes : pour dire qu'il s'agit de subordonnées, il faudrait en effet qu'elles lient une trace interne à l'apodose, ce qui ne correspond nullement à l'analyse adoptée ici) ${ }^{18}$. Toutes les circonstances sont donc réunies pour que la contribution de telles structures à l'interprétation de $\pi$ (et donc au sens global de la PC), si l'élément $k w$ - qu'elles contiennent est interrogatif, possède cette exhaustivité ou maximalité comme propriété supplémentaire ${ }^{19}$. 


\section{4.}

En d'autres termes, avec un $k w$ - interrogatif, l'apport de la protase serait comme en (25) :

\begin{tabular}{|l|l|l|}
\hline$(25)$ & $\mathrm{a}$ & $k{ }^{-I N T}{ }_{i}\left(\mathrm{C}^{\circ}[-\mathrm{Rad},+\mathrm{Qu}]\right)\left[{ }_{\mathrm{IP}} \ldots t_{i} \ldots\right]$ \\
\hline & $\mathrm{b}$ & $\Rightarrow>\lambda x\left[\operatorname{MAX}^{*}(x) \&[\phi(x)]\right.$ \\
\hline
\end{tabular}

Que signifie donc « $\operatorname{MAX}^{*}(x) »$ ici? Tout d'abord, que l'on ne s'intéresse plus seulement aux individus atomiques, mais aussi aux individus pluriels ou sommes individuelles. Soit un ensemble $\mathrm{E}$ contenant trois éléments, (26). Dans la méréologie issue des travaux de Lesniewski, ${ }^{20}$ les éléments de $\mathrm{E}$ sont aussi ses atomes :

(26) $E=\{a, b, c\}$

Outre l'ensemble $\mathrm{P}(\mathrm{E})$ des parties de $\mathrm{E}$, donné en (27), on peut, à la suite de Jacobson (1995) construire l'ensemble des individus strictement pluriels de $\mathrm{E}: \mathrm{E}^{++},(28 \mathrm{a})$, l'ensemble de ses individus atomiques ou pluriels : $\mathrm{E}^{+},(28 \mathrm{~b})$, et l'ensemble des individus comprenant, outre les éléments de $\mathrm{E}+, \varnothing: \mathrm{E}^{*},(28 \mathrm{c})$.

(27) $P(E)=\{\varnothing,\{a\},\{b\},\{c\},\{a, b\},\{a, c\},\{b, c\},\{a, b, c\}\}$

\begin{tabular}{|l|l|l|l|}
\hline$(28)$ & $\mathrm{a}$ & $\mathrm{E}^{++}$ & $=\{a+b, a+c, b+c, a+b+c\}$ \\
\hline & $\mathrm{b}$ & $\mathrm{E}^{+}$ & $=\{a, b, c, a+b, a+c, b+c, a+b+c\}$ \\
\hline & $\mathrm{c}$ & $\mathrm{E}^{*}$ & $=\{\varnothing, a, b, c, a+b, a+c, b+c, a+b+c\}$ \\
\hline & $\mathrm{d}$ & $E^{* *}$ & $=\{\varnothing, a+b, a+c, b+c, a+b+c\}^{21}$ \\
\hline
\end{tabular}

40 C'est $\mathrm{E}^{*}$ qui nous intéresse ici. La différence essentielle entre $\mathrm{E}^{*}$ et $\mathrm{P}(\mathrm{E})$ est la relation transitive, dite relation d'ingrédience, qu'on peut établir entre les individus, et qui est strictement interdite, par contre, entre par ex. $a,\{a, b\}$, et $\{a, b, c\}:$ l'atome a est un ingrédient de $a+b$, qui est un ingrédient de $a+b+c$, et par voie de conséquence, a est un ingrédient de $a+b+c$, ce que note (29) :

(29) $a \leq a+b \leq a+b+c$

41 A partir de là, un individu maximal est l'individu qui contient le plus grand nombre d'individus atomiques, $a+b+c$ dans notre exemple. 
Mais il faut se souvenir de ce que (25b) décrit une propriété (en intension), et donc un ensemble (en extension). De là suivent deux conséquences. D'une part, cet ensemble sera toujours un singleton, ce qui est en fait trivial, et, en fait, nullement une gêne. Mais d'autre part et surtout, si MAX* désigne la propriété pour un objet d'être un individu maximal défini sur $E^{*}\left(\mathrm{MAX}^{+}\right.$serait défini sur $\mathrm{E}^{+}$etc.), on a la garantie de ce que l'ensemble désigné par (25b) ne sera jamais vide, car si E lui-même est vide, $E^{*}$ contiendra $\emptyset$, cf. (28c).

Pensons maintenant aux dictons russes ou aux PC basques, qui ont typiquement le statut de lois. Logico-sémantiquement, on est dans un cas analogue à celui de conditionnelles comme (30a), dont la forme logique est donnée en $(30 b)^{22}$ : si aucun individu ne satisfait la conditionnante, la loi reste trivialement vraie.

\begin{tabular}{|l||ll|}
\hline$(30)$ & a & si quelqu'un fait ça, il sera puni \\
\hline & b & $\forall x[[$ hum $(x) \& f a i r e(x, c ̧ a)]:[$ être-puni $(x)]]$ \\
\hline
\end{tabular}

Et il en va de même des PC russes ou basques, comme on vient de le dire ${ }^{23}$.

\section{5 .}

Revenons maintenant aux corrélatives latines. On a vu qu'elles étaient sémantiquement de deux types, génériques-universelles ou spécifiques-définies, selon le temps. Comment concilier ce fait avec l'hypothèse que la protase n'apporterait qu'une propriété ?

le temps de l'apodose est épisodique, comme dans (2), l'interprétation du corrélat $\pi$ est naturellement celle proposée en (10). Dans ce cas, si l'interlocuteur n'a pas écrit de lettre, la proposition logique correspondant à la phrase sera fausse ou ininterprétable, selon que l'on préfère donner un interprétation russellienne, ou strawsonnienne, à la description définie non satisfaite que représente $\pi$. (Noter le contraste par rapport à ce qui vient d'être dit des PC basques ou russes.)

Par ailleurs, quand le temps est générique ou interprétable comme tel, un tout autre mécanisme d'interprétation se déclenche, celui dit de liage non-sélectif (v. Kamp 1984), qui consiste à lier toute les variables (qui ne sont pas déjà liées par un autre opérateur) par un opérateur de généricité (légitimé par le temps) : c'est précisément ce type de mécanisme qui permet de donner à (30a) son interprétation (b), les deux pronoms quelqu'un et il étant traités comme des variables ${ }^{24}$. Dans le cas qui nous concerne, l'opérateur de généricité va donc lier et le relatif et le corrélat, comme ci-après :

\begin{tabular}{|l|l|}
\hline (31) & qui $\mathrm{V}_{1}$ is $/$ hic $\mathrm{V}_{2}=>$ \\
\hline & GÉ $_{x}\left[\left[\mathrm{~V}_{1}(x)\right]:\left[\mathrm{V}_{2}(x)\right]\right]$ \\
\hline
\end{tabular}

Ainsi, si l'on admet que la virgule du texte latin de (19) marque le décrochage de la protase, et qu'il y a donc un pronom phoniquement zéro dans l'apodose (on aurait pu tout aussi bien avoir hic ou is), l'interprétation de cette phrase sera (32a) pour sa partie pertinente, et celle de (6), (32b) : 


\begin{tabular}{|l|l|l|}
\hline$(32)$ & $\mathrm{a}$ & GÉN $_{x}[[\operatorname{avoir}(x$, oreilles $)]:[\operatorname{entendre}(x)]]$ \\
\hline & $\mathrm{b}$ & GÉN $_{x}[[\operatorname{endroit}(x) \&$ être-en $(\operatorname{tyran}, x)]: \neg[$ être-en $($ république,$x)]]$ \\
\hline
\end{tabular}

C'est, en l'absence de données opposées, le traitement qu'on appliquera également au bambara.

\section{6 .}

On vient implicitement de reconnaître que différentes stratégies peuvent être employées pour " décoder » un sens plus ou moins identique, cf. le traitement distinct des versions allemande et latine de (19). Mais les possibilités de variation sont plus vastes encore. Considérons le cas du hindi. Si des ex. comme celui de la note 10 n'y sont pas rares, la double stratégie décrite pour le latin est disponible. Mais si, comme cela a été dit en 4.4, ils sont marginaux, il faut traiter la séquence initiale, dite protase jusqu'ici, comme n'étant que la partie visible d'une véritable relative libre topicalisée. Dans ce cas, ce qui est adjoint à la proposition radicale, ce n'est pas une proposition, mais un DP à tête phonétiquement non réalisée, comme dans l'analyse désormais classique des relatives libres anglaises (v. Groos \& van Riesmdijk 1981):

(33) $\left[{ }_{\mathrm{DP}} \varnothing\left[{ }_{\mathrm{CP}} \text { who }_{i}\left[\mathrm{IP} \text { you saw } t_{\mathrm{i}}\right]\right]\right]^{25}$

51 Cette analyse, qui simplifie l'interface syntaxe-sémantique du hindi (v. 4.4) est d'autant plus naturelle que cette langue n'a pas d'article défini: le zéro initial se conçoit alors aisément comme marqué [+spécifique].

Qui plus est, elle semble pouvoir s'adapter directement aux tours en der ... der de l'allemand archaïsant; certaines grammaires scolaires proposent en effet de l'analyser comme résultant de l'effacement d'un déterminant défini, l'élément barré en petites capitales de l'ex. ci-dessous (v. (21)), qui correspond exactement au $\emptyset$ de (33) :

(34) [ [DP DER [CP der ... die Hand ... taucht]], der wird...

\section{1 .}

Récapitulons. Derrière la formule (1) du début se cachent probablement deux structures syntaxiques très différentes, puisque le $\mathrm{CP}$ en $k w$-peut en fait être précédé d'un déterminant zéro, tête d'une expression nominale «DP ». Et lorsque ce $k w$ - est effectivement initial, il peut être interprété (non-conventionnellement) comme un interrogatif plutôt qu'un relatif; dans le premier cas, c'est cette propriété qui détermine, pour des raisons indépendamment justifiées, l'interprétation exhaustive ou maximale que l'on observe pour les PC basques ou russes; par contre, lorsqu'il s'agit bien d'un relatif, deux interprétations sont disponibles, liées à la variabilité sémantique des pronoms 
corrélats: l'une, spécifique, se tire de la traduction du corrélat comme dét. défini superficiellement intransitif, cf. (10), la protase relative apportant simplement une valeur pour la variable de prédicat laissée libre dans la traduction du pronom; l'autre enfin, générique, s'obtient grâce au liage non sélectif $d u k w$-initial et du corrélat par un opérateur de généricité lui-même rendu licite par le temps de l'apodose. On peut donc conclure qu'il n'y a pas de construction(s) corrélative(s) spécifique(s), du moins comme signes saussuriens, car l'interprétation des expressions examinées dans les langues les plus diverses se laisse ramener à un calcul strictement compositionnel. ${ }^{26}$

\section{2.}

54 Se pose encore une double question: (i) même si la sémantique des PC est régulière, pourquoi certaines langues possèdent-elles ces tournures, et d'autre pas? - et (ii) quel est le statut typologique du ou des paramètres qui interviennent?

On peut aisément répondre à (ii) ; en effet, seuls les dialectes basques parlés en France (et le haut-navarrais, parlé de l'autre côté de la frontière), "possèdent des corrélatives ». Mais je ne vois pas comment ce qui permet l'émergence de PC dans ces seuls dialectes les rapprocherait en quoi que ce soit du russe, et les différencierait corrélativement des dialectes guipuzcoan et biscayen parlés en Espagne. En d'autres termes, la possession ou non-possession des PC ne me semble en rien être une propriété typologique, si cette expression doit avoir un sens quelconque.

\section{3.}

Reste donc la question (i) : quel peut bien être le paramètre qui différencie ainsi les dialectes basques du nord de ceux du sud, ou encore le latin (ou l'allemand) du français ? L'hypothèse que je vais esquisser ici (et qui mériterait des recherches approfondies) m'a été inspirée par le constat que dans certaines PC, un élément de " coordination » semble introduire l'apodose, cf. le $i$ du russe en (8), (12) et (14), le eta du basque en (9) ${ }^{27}$, le $n$ - du hittite en (3) ${ }^{28}$ ou encore le sah gotique, illustré en (35), dont Mossé (1969: 103) dit que "c'est le pronom sa renforcé par la particule [...](u)h= latin que $»^{29}$

\begin{tabular}{|l|l|l|l|l|l|l|}
\hline (35) & sa.ei & $n u$ & ga.tairio & aina & anabusne & $\ldots$ \\
\hline & qui & donc & abolit & un & commandement & \\
\hline & & & & & & \\
\hline & sa.h & minnista & haitada & in & fiudangardjai & $\begin{array}{l}\text { himine (Mt } \\
5,19)\end{array}$ \\
\hline & celui-ci & plus-petit & sera-appelé & dans & palais-royal & céleste \\
\hline & $\begin{array}{l}\text { 'Qui aura violé le plus petit de ces commandements, celui-ci sera appelé petit dans le } \\
\text { royaume des cieux'30 }\end{array}$ \\
\hline
\end{tabular}


Bien sûr, il ne s'agit pas de considérer les deux propositions comme coordonnées. Pourrait-il s'agir d'un adverbe ayant la valeur de 'aussi' ? Cette hypothèse est attractive, car 'aussi' est une trad. fréquente de russe $i$; mais quelle en serait la portée? Certainement pas le corrélat $\pi$, puisqu'il n'apparaît pas comme s'ajoutant à d'autres individus dont on prédiquerait la même propriété; pourrait-il s'agir d'un adverbe renforçant le fait que le prédicat de l'apodose est, par la présence même de la protase, un second prédicat attribué au sujet? Une première objection est fournie par un dicton comme (36) :

\begin{tabular}{|l|l|l|l|l|l|}
\hline (38) & čto & budet, & to & i & budet \\
\hline & quoi & sera & ça & et & sera \\
\hline \multicolumn{4}{|l}{ 'Ce qui sera sera' } \\
\hline
\end{tabular}

Puisque le prédicat est répété, $\boldsymbol{i}$ ne peut avoir le sens de « aussi ». La seconde objection est plus forte: c'est la position même qu'occupe cette particule; ainsi, en basque, si l'on devait modifier le second prédicat par 'aussi', on lui suffixerait ere, ce qui est impossible dans le contexte des PC.

Reste donc l'hypothèse que ces mots occupent une position structurale particulière. Deux possibilités sont envisageables. D'une part, ils pourraient être la matérialisation (l'épel) d'un trait que seules les langues à $\mathrm{PC}$ posséderaient, et qui se situerait dans le $\mathrm{C}^{\circ}$ de la phrase radicale. La structure syntaxique de (38) serait alors :

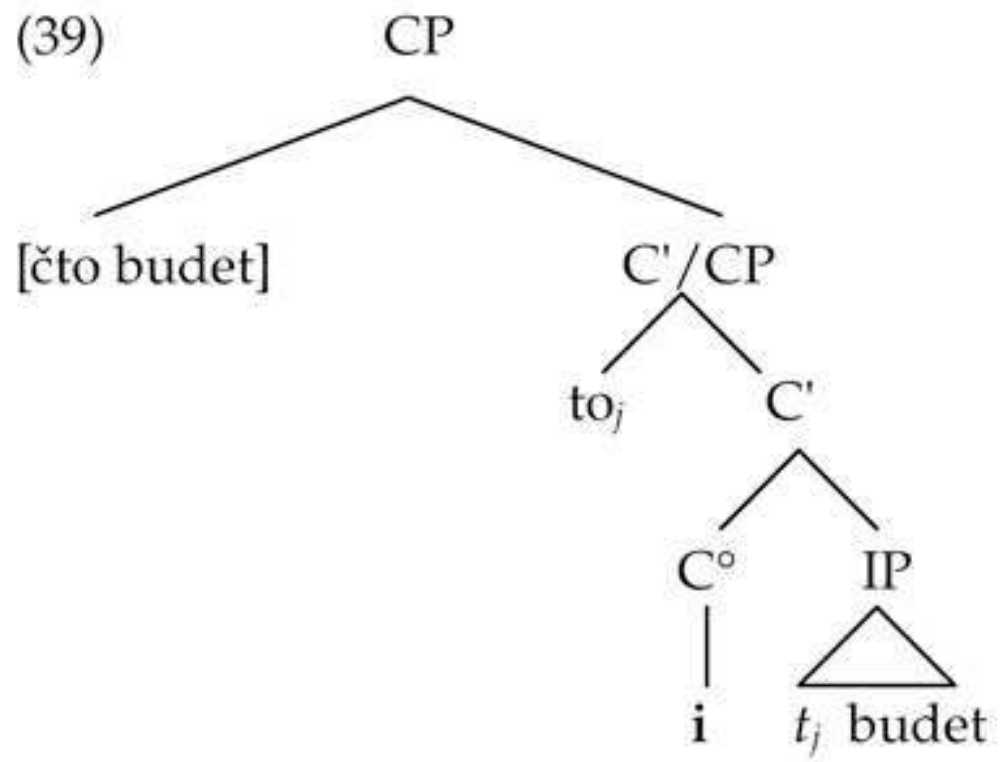

Noter que la duplication de $\mathrm{C}^{\prime}$ ou $\mathrm{CP}$ est nécessaire, si ce trait associé à $\mathrm{C}^{\circ}$ est précisément ce qui légitime la présence de la protase, et que la focalisation du corrélat se fait par adjonction à C' ou par montée en Spéc,CP.

61 L'autre hypothèse consisterait à prendre $i$, eta etc. comme des têtes fonctionnelles spécifiques, ou peut-être plus simplement comme instanciant un type non reconnu de « connecteur ». Dans ce cas, la protase des PC serait le spécificateur de la projection de 
cette tête, et l'apodose, son complément ${ }^{31}$ (voir à cet égard la présentation par Progovac 1998 des théories récentes qui proposent de même que la conjonction de coordination soit la tête structurale de syntagmes coordonnés). La «possession des phrases corrélatives " se ramènerait dans le premier cas à un trait, et dans le second à la possession d'une tête fonctionnelle, dont ne disposeraient pas les autres langues ou dialectes. $^{32}$

\section{BIBLIOGRAPHIE}

BIzos, M. (1966) Syntaxe grecque. Paris : Vuibert.

comorovsKI, I. (1995) Interrogative Phrases and the Syntax-Semantics Interface. Dordrecht : Kluwer.

COOPER, R. (1979) The Interpretation of Pronouns. In F. Heny \& H. Schnelle (eds.), Syntax \&

Semantics 10 (New York : Academic Press), 61-92.

DAYAL, V. (1996) Locality in Wh Quantification. Dordrecht : Kluwer.

DEBEAUVAIS, L. et al. (1962) Cours de langue latine. Paris : Belin.

GASON, J. et al. (1984) Abrégé de grammaire latine. Paris : Magnard.

GROOS, A. \& VAN RIEMSDIJK, H. (1981) Matching Effetcs in Free Relatives : A Parameter of Core

Grammar. In A. Belletti et al. (eds.), Theory of Markedness in Generative Grammar (Pise : Scuola Normale Superiore), 171-216.

Grosu, A. [à par.] The Semantic Diversity of Internally-headed Relative Clauses. Ms., Univ. de TelAviv.

GUPTA, S. (1986) Discourse Grammar of Hindi. A Study in Relative Clauses. New Delhi : Bahri Publications.

HAUDRY, J. (1973) Parataxe, hypotaxe et corrélation dans la phrase latine. BSL 68/1, 147-186.

IZVORSKI, R. (1996) The Syntax and Semantics of Correlative Proforms. NELS 26, 133-147.

JACOBSON, P. (1995) On the Quantificational Force of English Free Relatives. In E. Bach et al. (eds.), Quantification in Natural Languages (Dordrecht : Kluwer), 451-486.

KAMP, H. (1984) A Theory of Truth and Semantic Representation. In J. Groenendijk et al. (eds.), Truth, Interpretation and Information (Dordrecht : Foris), 1-43.

LINK, G. (1983) The Logical Analysis of Plural and Mass Terms. In R. Bäuerle et al. (eds.), Meaning, Use and Interpretation (Berlin : de Gruyter), 303-323.

Mossé, Fernand. [1942] (1969) Manuel de la langue gotique. Paris : Aubier.

PROGOVAC, L. (1998) Structures for Coordination I \& II. GLOT International 3/7, 3-6 \& 3/8, 3-9.

REBUSCHI, G. (1998a) E-Type Pronouns and Quantification in a Basque Dialect. Com. au Colloque de sémantique (de préférence non-lexicale), Univ. Paris 7, mai.

(1998b) 'Nouvelles remarques sur haina'. Lapurdum 3, 53-75. 
ZRIBI-HERTZ, A., \& HANNE, J.-F. (1995) Pronoms, déterminants et relatives en bambara de Bamako.

Linguistique africaine 15, 91-135.

\section{NOTES}

1. Je remercie le public du colloque de Nanterre (dont en particulier D. Creissels, A. Montaut et A. Rousseau) pour leurs remarques sur l'exposé de novembre 1998, ainsi que P. Cabredo-Hofherr, K. Ferret, A. Grosu, J.-L. Léonard, S. Sauvageot, E. Soare, H. van Riemsdijk et mes étudiants de DEA et en thèse à Paris 3 pour leurs commentaires et discussions sur divers points soulevés ici.

2. Même si l'on admet que ces agencements (qui ne sont réductibles ni au signifié ni au signifiant des entités qui entrent dans leur composition), ne sont pas la pure expression de la $3^{e}$ face, proprement grammaticale, des signes linguistiques, mais dépendent aussi de contraintes syntaxiques qui leur sont externes (comme la théorie « X-barre » par exemple).

3. Le fait que (4) présente une variante dans laquelle le nom est répété dans les deux propositions indique que, dans cette langue au moins, l'analyse traditionnelle par mouvement (v. la citation de Bizos supra) n'est pas tenable :

\begin{tabular}{|c|c|c|c|c|c|}
\hline $\begin{array}{l}\text { jo } \\
\text { quelle fille }\end{array}$ & $\begin{array}{l}\text { larkii } \\
\text { debout }\end{array}$ & $\begin{array}{l}\text { khaRii } \\
\text { est }\end{array}$ & hai, & $\begin{array}{l}\text { vo } \\
\text { cette }\end{array}$ & $\begin{array}{l}\text { laRkii } \\
\text { fille }\end{array}$ \\
\hline
\end{tabular}

4. Les dictons russes du texte sont tirés de V. Anikine (ed.), 1988: Russkie poslovitsy i govorki ,Moscou : Xudožestvennaja Literatura. Je remercie André Salem de me l'avoir procuré, ainsi que de m'avoir signalé la grande fréquence de $i$, conjonction sur laquelle je reviendrai en 6.3.

5. Trad. de L. Léon (1947) : Jesu-Kristo gure Jaunaren Ebanjelio Saindua. Ustaritz : chez l'auteur.

6. Qu'il soit clair que pour moi, en dépit des apparences, une phrase comme (7) n'a pas la même structure que qui vivra verra ou (ii) je respecte qui me respecte, puisque l'on y trouve une relative libre en position argumentale (dans la position structurale du sujet ou de l'objet), plutôt qu'à l'extérieur du IP noyau il se dort cuntre tere de (7).

7. L'opérateur $\lambda$ qui lie $Q$ dans la formule proposée permet par ailleurs de combiner $\pi$ avec le prédicat fourni par l'apodose, soit resp. la propriété d'avoir été vu par moi, ou celle d'être aimé... Dans le cas de l'ex. hindi suivant (Dayal 1996 : 153), il faut admettre que la restriction $R$ de (10) se décompose en deux propriétés $R_{1} \& R_{2}$, dont l'une est fixée par laRkii 'fille' et l'autre est libre :

$\begin{array}{llllll}\text { (i) jo vahaan khaRii hai Raam us laRkit-ko jaantaa hai } \\ \text { kw- là } & \text { debout est Ram cette fille } & \text { connaît Aux }\end{array}$

'Ram connaît la fille qui est debout là'

8. Comme l'a montré Comorovski (1995), la quantification universelle qui traduit le premier de deux éléments $k w$ - n'est qu'apparente : il ne peut s'agir en fait que d'une quantification restreinte à un ensemble fini, donné dans le discours.

9. (D)etchepare, B. [1545] 1980. Linguae vasconum primitiae; éd. critique par P. Altuna, Bilbao : Mensajero, 1980.

10. Dayal n'indique pas pourquoi le marqueur de génitif change.

11. Ce tour, encore productif aujourd'hui, est attesté dès le $18^{\mathrm{e}}$, v. Rebuschi (1998b).

12. Voir plus loin sur ceci.

13. Le statut d'ex. comme (i) (Dayal 1995 : 185), d'interprétation nécessairement non-spécifique, devient cependant étonnant :

(i) jo aadmii sabhii bhaashaayeN bol sake vo paidaa nahii huaa
quel homme tout langues parler peut lui né 'l'homme qui peut parler toutes les langues n'est pas [encore] né' 
14. Le fait que les tours en der...der n'aient pas été ambigus est peut-être lié au fait que les deux constructions coexistaient (une autre explication sera évoquée en 5.6). Henk van Riemsdijk me signale qu'en allemand parlé d'aujourd'hui, la disparition des tours en der...der a eu pour effet de permettre parfois une interprétation définie pour ceux en wer...der, ce qui s'explique très bien par la notion de quantification (universelle) dégénérée (car ne portant que sur un singleton).

15. Die Heilige Schrift, Genève : Genfer Bibelgesellschaft, 1985.

16. Voici la traduction anglaise par Ogden, qui reproduit le même schéma: Whereofone cannot speak, thereof one must be silent. Merci à David Bundy pour ces deux exemples.

17. Noter que le latin offre donc aussi deux PC pour (19) et (21), avec un corrélat phoniquement zéro dans le premier cas, mais, crucialement, le même relatifQuI dans chaque protase.

18. Pour le russe, il manque par ailleurs un morphème abstrait d'interrogation, réalisé prosodiquement, pour faire des protases des phrases radicales. En tout état de cause, je suis en désaccord complet avec Haudry (1973: 152) qui affirme que «les deux parties [...] sont grammaticalement indépendantes l'une de l'autre ».

19. L'interprétation d'un $\mathrm{CP}$ à tête $\mathrm{C}^{\circ}[-\mathrm{Rad},+\mathrm{Qu}]$ (pour Radical et Question) comme question indirecte n'est donc possible que si ce $\mathrm{CP}$ est de plus régi ou gouverné, ce qui n'est pas le cas ici, on vient de le voir.

20. V. Link 1983.

21. Cet ensemble se manifeste dans des exemples non-standard comme J'ai vu exactement zéro chevaux / je n'ai vu aucun chevaux (attesté depuis le Moyen français).

22. J'écris bien "forme logique " sans majuscules, car il s'agit d'une représentation vériconditionnelle (partielle), et non d'un niveau de représentation « syntaxique » abstrait.

23. L'exhaustivité peut bien sûr être donnée directement par les morphèmes eux-mêmes, comme dans le texte latin de (9).

24. En fait, l'opérateur de généricité et le quantificateur universel ne sont pas strictement équivalents, puisque le premier tolère des exceptions, mais cette distinction est sans pertinence ici.

25. Selon Jacobson (1995), l'ambiguïté de ces exemples peut se résoudre en posant qu'on fait référence à un individu maximal, qui peut correspondre à un individu pluriel (interpr. générique) ou atomique (interpr. spécifique). Par ailleurs, il est indifférent à mon propos de savoir si le CP est le complément du $\mathrm{D}^{\circ}$ vide, ou s'il y a un nœud NP/SN intermédiaire.

26. V. Grosu (à par.) pour des résultats du même ordre obtenus dans un domaine voisin.

27. Et en (16), que j'avoue être pour l'heure incapable d'analyser.

28. V. Haudry (1973: 181) : «l'anaphorique $[=\pi]$ hittite est toujours précédé d'une particule de phrase, $n u$ - dans les textes classiques... ».

29. Se rappeler que l'enclitique latin -que marque la coordination! Quant à l'élément initial sa-ei c'est bien un relatif obtenu par suffixation de -ei au démonstratif proche sa.

30. La « King James' Version» donne aussi une PC pour ce passage : Whosoever shall break one of these least commandments ... he shall be called the least in the kingdom of heaven.

31. V. encore la position du connecteur alors, en tête des apodoses conditionnelles, qui rend licite une protase conditionnante. Cette hypothèse, plus tentante que la première, fait cependant difficulté en russe, où le(s) corrélat(s) précède(nt)i.

32. Comme cette tête est effectivement matérialisée parfois, je ne trouve pas gênant de poser qu'elle existe aussi en allemand par ex., mais sans face phonique réalisable. 


\section{AUTEUR}

GEORGES REBUSCHI

TYGRE -Université Paris III 